\section{Cureus}

Received 04/07/2017

Review began 04/14/2017

Review ended 04/14/2017

Published 04/18/2017

C) Copyright 2017

Mangi et al. This is an open access article distributed under the terms of the Creative Commons Attribution License CC-BY 3.0., which permits unrestricted use, distribution, and reproduction in any medium, provided the original author and source are credited.

\title{
Ambulatory Heart Failure Monitoring: A Systemic Review
}

\author{
Muhammad A. Mangi ${ }^{1}$, Hiba Rehman ${ }^{1}$, Muhammad Rafique ${ }^{2}$, Michael Illovsky ${ }^{3}$ \\ 1. GME Internal Medicine, Orange Park Medical Center 2. Anesthesiology, Liaquat National Hospital 3. \\ Cardiology, Orange Park Medical Center
}

$\square$ Corresponding author: Muhammad A. Mangi,drmasif33@yahoo.com

Disclosures can be found in Additional Information at the end of the article

\section{Abstract}

Heart failure (HF) is one of the leading causes of morbidity and mortality and has a large effect on the country's economy. Although there have been major advances in HF monitoring, including more advanced pharmacological management and device-based therapy, HF-related mortality remains high. It is important to monitor HF so that HF-related hospitalization and mortality can be prevented. Due to the lower sensitivity of clinical features and biochemical markers, as well as the failure of telemonitoring in early detection of HF, more advanced techniques have been sought to more accurately predict impending HF, in order to address timely pharmacological management and prevent heart failure hospitalization (HFH). Devicebased therapy has passed through various stages and culminated in the recently introduced CardioMEMS $^{\mathrm{TM}}$ (St. Jude Medical, Inc., Saint Paul, Minnesota). CardioMEMS ${ }^{\mathrm{TM}}$ is a wireless pulmonary artery pressure (PAP) monitoring device, which continuously monitors PAP and transmits data to a healthcare provider. It rapidly identifies changes in intracardiac pressure and allows timely pharmacological management. CardioMEMS ${ }^{\mathrm{TM}}$ showed a higher reduction of HFH compared to any other devices.

Categories: Internal Medicine, Cardiology, Preventive Medicine

Keywords: cardiac implantable electronic device, heart failure hospitalisation, heart failure monitoring

\section{Introduction And Background}

Heart failure (HF) is a clinical syndrome characterized by the inability of the heart to meet the metabolic demand of the body either by left ventricular dysfunction or right ventricular (RV) dysfunction. The prevalence is 5.8 million in the US and over 23 million worldwide [1]. The incidence is continuously increasing and reaching 650,000 people per year with the financial burden of 30.7 billion dollars [2-3]. Despite the increasing technology of effective pharmacotherapy and device-based therapies for the management of HF, the recurrent hospitalization, morbidity, and mortality remain high [4]. Each admission of acute decompensated HF is the predictor of recurrent admission; i.e., 30\% in one month and 50\% in six months $[1,3]$. Most of these admissions are due to the progressive rise in intracardiac filling pressure independent of ejection fraction (EF) and etiology [5-9].

Implantable hemodynamic monitoring device such as CardioMEMS ${ }^{\mathrm{TM}}$ (St. Jude Medical, Inc., Saint Paul, Minnesota) detects cardiac filling pressure. These devices detect rising cardiac filling pressure days/weeks before the symptoms, allowing the healthcare provider to intervene in order to prevent HF hospitalizations [10]. Several methods have been tried to reduce heart failure hospitalization (HFH) including weight monitoring, clinical features, biomarkers, and device-based diagnostics, but were not effective in reducing HFH [11-14]. Pulmonary artery 


\section{Cureus}

pressure (PAP) monitoring has been seen to be effective in reducing $\mathrm{HFH}$ in the

“CardioMEMS ${ }^{\mathrm{TM}}$ Heart Sensor Allows Monitoring of Pressure to Improve Outcomes in New York Heart Association (NYHA) functional Class III Heart Failure Patients” (CHAMPIONS) trial [15]. These effects were persistent in both HF with reduced EF (HFrEF) and HF with preserved EF (HFpEF) [16]. HFH was positively correlated to increasing PAP and decreased with PAP guideline-directed therapy [17]. Hence in 2014, United States Food and Drug Administration (FDA) approved the usage of CardioMEMS ${ }^{\mathrm{TM}}$ in the patient with chronic HF, NYHA functional class III to reduce HFH [18].

\section{Review}

\section{Common methods for HF monitoring}

History and Physical

The history and physical examination often help in the diagnosis of HF. The patient often presents with shortness of breath, paroxysmal nocturnal dyspnea, orthopnea, crackles, third heart sound, jugular venous distension, weight gain, and edema, but these features have limited sensitivity and are considered a late manifestation of HF (Table 1). Using symptoms as markers of HF has limited effect on reducing HFH [19]. This management basically involves patient self-assessment and management, including diuretic dose adjustment, which is often an issue in non-compliant and poor self-care skill patients [19]. Telemonitoring has been emerging field in medicine but has failed to show benefit in HFH and mortality [19].

History

Dyspnea

Paroxysmal nocturnal dyspnea

Orthopnea

Cough

Weight gain

\section{Physical}

Jugular venous distension

Hepatojugular reflex positive

Lung crackles

S3 heart sound

Edema

\section{TABLE 1: History and physical examination features for heart failure monitoring}

Biochemical Markers

Use of biochemical markers to reduced HFH is still under consideration, but data is still under debate. There is no specific threshold level for these markers. Initially, Lainchbury, et al. compared N-terminal pro-B-type natriuretic peptide (NT-pro-BNP) guided medical therapy with clinically guided medical therapy and usual care, and interestingly found decreased HFrelated mortality in patient with NT-pro-BNP guided medical therapy [20]. This benefit was seen in a subgroup of patient $\leqslant 75$ years old. However, this study did not show any reduction in HFH. McQuade, et al. systemic review data supported the mortality benefit of NT-pro-BNP but also stated that when NT-pro-BNP target is achieved, it decreases HFH [21]. McQuade, et al. data had a specific threshold of NT-pro-BNP (decrease of at least 30\%) and BNP $(250 \mathrm{pg} / \mathrm{ml}$ or less). It was low-strength evidence that stated an association exists between achieving NP predischarge thresholds and reduced HF mortality and readmission. Additionally, Huang, et al. 


\section{Cureus}

proposed NT-pro-BNP based score which also predicts hospital mortality in HF patients [22]. Another study monitored plasma BNP level in chronic stable HF patient in the ambulatory setting to predict inevitable decompensation and found both asymptomatic and symptomatic HF patient had a wide range of plasma BNP levels [23].

\section{Echocardiography}

With further advancement in the medical sciences, echocardiography has been used in the management of HF in order to prevent HFH. It has its own drawback of cost, intra-observer variability, quality of the echocardiographic picture, patient anatomical characteristics, obesity, and accuracy of the test.

\section{Right Heart Catheterization}

Right heart catheterization (RHC) has been increasingly used in measuring filling pressure and volume status of the patient, which provide indispensable information in guiding medical therapy in HF patient. However, its utility is limited due to invasive nature and risk of the procedure (Table 2).

\section{Other common methods for heart failure monitoring}

\section{Telemonitoring}

NT -pro-BNP

BNP

Chest radiograph

Echocardiography

Right heart catheterization

\section{TABLE 2: Other methods for heart failure monitoring}

NT-pro-BNP: N-terminal pro-B-type natriuretic peptide. BNP: B-type natriuretic peptide.

\section{Cardiac implanted electronic devices for heart failure monitoring}

Cardiac implanted electronic devices (CIEDs) are being evaluated to provide ambulatory monitoring of HF. These devices are a closed system consisting of a "sensor" to sense the response and an "effector" to initiate a response. The sensor can take different forms, including a patient, a provider, and/or a device with each form having its own advantage and disadvantage. The main advantage of a device includes continuous monitoring, objective metrics without bias and provides patient specific information (Table 3). 


\section{Cureus}

Electrophysiological sensors

\section{Hemodynamic sensors}

Chronicle (right ventricular pressure)

ePOD (estimated pulmonary artery diastolic pressure)

Heart PAD (left atrial pressure)

CardioMEMS $^{\mathrm{TM}}$ (pulmonary artery pressure)

\section{TABLE 3: Cardiac implanted electronic devices for heart failure monitoring}

SDAAM: Standard deviation of 5-minute median atrial-atrial intervals. SDANN: Standard deviation of 5-minute median ventricular intervals.

Electrophysiological Sensors

Most commonly used CIEDs are pacemaker and defibrillator. They have the capability of sensing certain atrial and ventricular arrhythmias. Through the closed-loop system, they sense and provide appropriate response either pacing or shock. Treating arrhythmias can have a stabilizing effect on HF. Monitoring other electrophysiological variables may have a role in predicting and preventing acute decompensated HF (ADHF). As increased mean heart rate has been demonstrated before ADHF, with a returned back to baseline once ADHF is treated [24]. Such heart rate variability (HRV) can be sensed by the implantable device and therapy initiated to prevent potential hospitalization. HRV can be measured from implantable devices with atrial leads by determining the standard deviation of 5-minute median atrial-atrial intervals (SDAAM) or consecutive ventricular (N-N) intervals (SDANN) over a 24-hour period. These devices detect HRV as early as three weeks before hospitalization [25]. The sensitivity of these devices was 70\% in predicting hospitalization.

\section{Hemodynamic Monitoring Sensors}

The main pathophysiology of ADHF is a rise in ventricular filling pressure, therefore the main focus of HF management is to lower the ventricular filling pressure without affecting cardiac output. Historically, an RHC was required to monitor hemodynamic status. This led to the development of implantable cardiac filling pressure monitoring devices. These devices have been shown to provide information almost comparable to an RHC [26]. Early work also demonstrated the safety and feasibility of long-term utilization of RV pressure monitoring [27]. More importantly estimated pulmonary artery diastolic (ePAD) pressure monitoring has a stronger correlation in HF as it is a closer surrogate of left atrial filling pressure [28]. Earlier data suggested continuous RV pressure monitoring reduces HFH [29]. Later, Chronicle Offers Management to Patients with Advanced Signs and Symptoms of Heart Failure (COMPASS-HF) trial (Medtronic Inc., Minneapolis, Minnesota) was designed to evaluate therapeutic choices based on RV pressure and its effect on HF hospitalization. However, it did not show any significant effect on hospitalization compared to control group [30]. Secondary analysis of the same study showed $36 \%$ relative risk reduction for first HFH. The possible reason of failure to achieve primary endpoint in this study was that the control group might have had a lower than expected number of event. This trial not only helped in reducing HFH but also gave us the insight into the pathophysiology of HF with both reduced and preserved EF. It demonstrated the baseline ventricular filling and ventricular relaxation between groups [10]. In both EF group, patients' ePAD was significantly higher before the episode of ADHF, but the number of 
advanced day notice was lower and ventricular filling pressure was significantly higher in patients with preserved EF compared to the patients with low EF. The Reducing

Decompensation Events Utilizing Intracardiac Pressures in Patients with Chronic Heart Failure (REDUCE HF trial) combines both devices, implantable hemodynamic monitoring device (IHM) and implantable cardioverter defibrillator to check for RV pressure guided medical therapy [31]. This device also did not show any satisfactory result on reducing HFH. The caveat of this study was, they used IHM device data as a guideline for treatment. Interestingly, continuous measurement of RV pressure is the most straightforward form of implanted hemodynamic monitoring, as we extrapolate pulmonary pressure from RV pressure. However, most patients with ADHF have pulmonary congestion secondary to elevated left atrial pressure (LAP) [32]. Therefore continuous monitoring of LAP may provide a better method of HF monitoring. This discussion continued and in 2010, Hemodynamically Guided Home Self-Therapy in Severe Heart Failure Patients (HOMEOSTASIS) trial demonstrated a reduction of HFH and mortality when treatment was guided on LAP [33]. In this trial, they used implantable Heart POD (St. Jude Medical Inc, Minneapolis, Minnesota). This study formed a basis of a new large populationbased ongoing LAPTOP-HF study designed to check the role of implantable left atrial pressure monitoring in association with guiding treatment on HF [34]. This might change the paradigm of HF management.

\section{CardioMEMS ${ }^{T M}$ Monitoring for $H F$}

CardioMEMS $^{\mathrm{TM}}$ is a wireless device that monitors PAP. It is implanted in the distal pulmonary artery via an RHC. It has been seen that PAP measured by CardioMEMS ${ }^{\mathrm{TM}}$ device correlates with PAP measured by Swan-Ganz and echocardiography [35]. The first time CardioMEMS ${ }^{\mathrm{TM}}$ was investigated was in the CHAMPIONS trial [36], a multicenter, single-blinded, randomized control trial of 550 patients with NYHA class III HF. The heart failure medications were adjusted on the basis of data generated by the sensor. The CHAMPIONS trial showed a promising result with $28 \%$ reduction of HF hospitalization in six months and $37 \%$ in 15 months without increasing other causes of hospitalization [37]. The CardioMEMS ${ }^{\mathrm{TM}}$ device worked well without any sensor failure and a lower complication rate. This is the first sensor-based device which demonstrated a significantly lower risk of HFH. Due to this success, in 2014 the FDA approved the use of CardioMEMS ${ }^{\mathrm{TM}}$ sensor implant in patients with HFpEF and HFrEF with NYHA class III on optimal medical therapy and a history of HFH within the last year [19]. The reduced number of hospitalization in CHAMPIONS trial was related to pressure monitoring via CardioMEMS $^{\mathrm{TM}}$ sensor implant which led to appropriate and timely management with dose adjusted diuretics [38]. Subgroup analysis of the study showed the promising result of HF management based on PAP sensor and was more reliable and effective in reducing HFH than HF management base on clinical signs alone [39]. During study follow-up at six months, the patient in the treatment group who had CardioMEMS ${ }^{\mathrm{TM}}$ sensor implant received maximal medical therapy including diuretics, vasodilators, and the neurohormonal antagonist [17]. Remote PAP monitoring guided medical therapy reduces hospitalization of the patient with HFrEF with and without cardiac resynchronized therapy (CRT) [40]. This provides additive benefit in CRT patient. There was a significant reduction in all-cause hospitalization and mortality [41]. Surprisingly, measuring PAP with a single RHC alone does not entirely rule out pulmonary hypertension as it underdiagnoses pulmonary hypertension related to left HF [42]. This evidence is further supported by another study in which the author compared HF patient who had implanted hemodynamic device (CardioMEMS ${ }^{\mathrm{TM}}$ ) with no implanted hemodynamic device and reported significant improvement of Kansas city cardiomyopathy questionnaire score and 6-minute walk test in the CardioMEMS ${ }^{\mathrm{TM}}$ group [43]. These findings are in favor of the CHAMPIONS trial which has high evidence of reduction of HFH and mortality. Additionally, a recently published retrospective study by Desai, et al. further intensifies the CHAMPIONS trial result [44]. There was a $45 \%$ reduction of $\mathrm{HFH}$ observed in this study compared to $28 \%$ in 


\section{Cureus}

CHAMPIONS trial. The reduction persisted even in later months. Concomitantly the reduced HFH was not associated with increased risk of all-cause hospitalization (Figure 1).

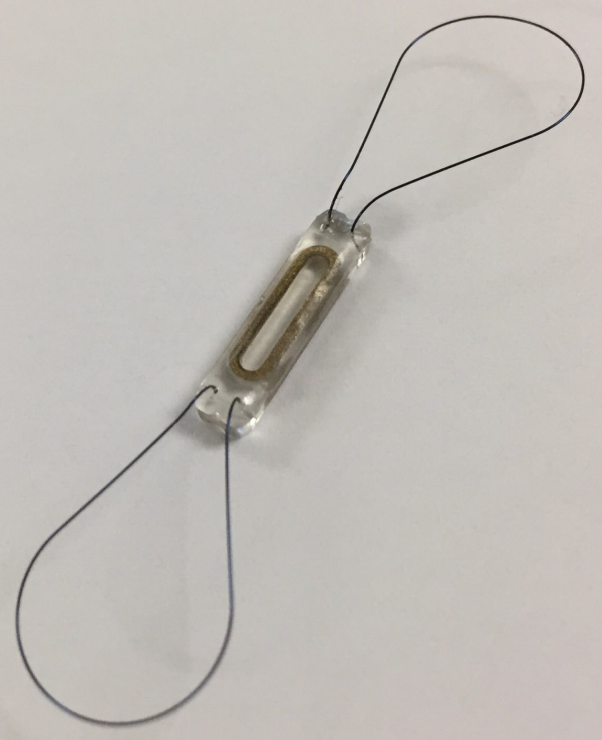

FIGURE 1: CardioMEMS device

Improving survival with modern therapy has resulted in HF patients living longer with left ventricular assist devices (LVADs). The author further did sub-analysis of CHAMPIONS trial in order to assess the validity of PAP-directed therapy on optimization of medications, pump parameters, and timing of heart transplantation in the patient receiving LVAD [45]. The data obtained from treatment group revealed more frequent medication adjustment than the control group.

\section{Conclusions}

HF continues to be a major public health problem with a significant financial burden on the country's economy. As advances in medicine lead to longer life expectancy, more reliable and valid methods will be required to appropriately intervene in order to prevent HF-related hospitalization and death. Implantable hemodynamic devices are the newly emerging tools in the field of HF management. CardioMEMS ${ }^{\mathrm{TM}}$ appears to be a leading innovation in the management of HF. However, given a small number of trials with small sample size, larger multicenter trials are required to make valid recommendations in order to reduce $\mathrm{HFH}$, improve the quality of life, and decrease morbidity and mortality related to HF.

\section{Additional Information}

\section{Disclosures}

Conflicts of interest: In compliance with the ICMJE uniform disclosure form, all authors declare the following: Payment/services info: All authors have declared that no financial 
support was received from any organization for the submitted work. Financial relationships: All authors have declared that they have no financial relationships at present or within the previous three years with any organizations that might have an interest in the submitted work. Other relationships: All authors have declared that there are no other relationships or activities that could appear to have influenced the submitted work.

\section{References}

1. Roger VL: Epidemiology of heart failure. Circ Res. 2013, 113:646-659. 10.1161/CIRCRESAHA.113.300268

2. Mozaffarian D, Benjamin EJ, Go AS, et al.: Heart Disease and Stroke Statistics-2016 update: a report from the American Heart Association. Circulation. 2016, 133:e38-e360.

10.1161/CIR.0000000000000350

3. Yancy CW, Jessup M, Bozkurt B, et al.: 2013 ACCF/AHA guideline for the management of heart failure: a report of the American College of Cardiology Foundation/American Heart Association Task Force on Practice Guidelines. J Am Coll Cardiol. 2013, 62:e147-e239. 10.1016/j.jacc.2013.05.019

4. Chun S, Tu JV, Wijeysundera HC, et al.: Lifetime analysis of hospitalizations and survival of patients newly admitted with heart failure. Circ Heart Fail. 2012, 5:414-421.

10.1161/CIRCHEARTFAILURE.111.964791

5. Cotter G, Metra M, Milo-Cotter O, et al.: Fluid overload in acute heart failure--re-distribution and other mechanisms beyond fluid accumulation. Eur J Heart Fail. 2008, 10:165-169. 10.1016/j.ejheart.2008.01.007

6. Gheorghiade M, Filippatos G, De Luca L, et al.: Congestion in acute heart failure syndromes: an essential target of evaluation and treatment. Am J Med. 2006, 119:S3-S10. 10.1016/j.amjmed.2006.09.011

7. Kato M, Stevenson LW, Palardy M, et al.: The worst symptom as defined by patients during heart failure hospitalization: implications for response to therapy. J Card Fail. 2012, 18:524533. 10.1016/j.cardfail.2012.04.012

8. Metra M, Dei Cas L, Bristow MR: The pathophysiology of acute heart failure--it is a lot about fluid accumulation. Am Heart J. 2008, 155:1-5. 10.1016/j.ahj.2007.10.011

9. Schiff GD, Fung S, Speroff T, et al.: Decompensated heart failure: symptoms, patterns of onset, and contributing factors. Am J Med. 2003, 114:625-630.

10. Zile MR, Bennett TD, St John Sutton M, et al.: Transition from chronic compensated to acute decompensated heart failure: pathophysiological insights obtained from continuous monitoring of intracardiac pressures. Circulation. 2008, 118:1433-1441. 10.1161/CIRCULATIONAHA.108.783910

11. Chaudhry SI, Mattera JA, Curtis JP, et al.: Telemonitoring in patients with heart failure . N Engl J Med. 2010, 363:2301-2309. 10.1056/NEJMoa1010029

12. Koehler F, Winkler S, Schieber M, et al.: Impact of remote telemedical management on mortality and hospitalizations in ambulatory patients with chronic heart failure: the telemedical interventional monitoring in heart failure study. Circulation. 2011, 123:18731880. 10.1161/CIRCULATIONAHA.111.018473

13. Ong MK, Romano PS, Edgington S, et al.: Effectiveness of remote patient monitoring after discharge of hospitalized patients with heart failure: the better effectiveness after transition - heart failure (BEAT-HF) randomized clinical trial. JAMA Intern Med. 2016, 176:310-318. 10.1001/jamainternmed.2015.7712

14. van Veldhuisen DJ, Braunschweig F, Conraads V, et al.: Intrathoracic impedance monitoring, audible patient alerts, and outcome in patients with heart failure. Circulation. 2011, 124:1719-1726. 10.1161/CIRCULATIONAHA.111.043042

15. Abraham WT, Stevenson LW, Bourge RC, et al.: Sustained efficacy of pulmonary artery pressure to guide adjustment of chronic heart failure therapy: complete follow-up results from the CHAMPION randomised trial. Lancet. 2016, 387:453-461. 10.1016/S01406736(15)00723-0

16. Adamson PB, Abraham WT, Bourge RC, et al.: Wireless pulmonary artery pressure monitoring guides management to reduce decompensation in heart failure with preserved ejection fraction. Circ Heart Fail. 2014, 7:935-944. 10.1161/CIRCHEARTFAILURE.113.001229 
17. Costanzo MR, Stevenson LW, Adamson PB, et al.: Interventions linked to decreased heart failure hospitalizations during ambulatory pulmonary artery pressure monitoring. JACC Heart Fail. 2016, 4:333-344. 10.1016/j.jchf.2015.11.011

18. US Food and Drug Administration Center for Devices and Radiological Health. CardioMEMSTM HF System approval letter. (2014). Accessed: March 2, 2017: https://www.accessdata.fda.gov/scripts/cdrh/cfdocs/cfpma/pma.cfm?id=P100045.

19. Lampert BC, Emani S: Remote hemodynamic monitoring for ambulatory left ventricular assist device patients. J Thorac Dis. 2015, 7:2165-2171. 10.3978/j.issn.2072-1439.2015.10.45

20. Lainchbury JG, Troughton RW, Strangman KM, et al.: N-terminal pro-B-type natriuretic peptide-guided treatment for chronic heart failure: results from the BATTLESCARRED (NTproBNP-Assisted Treatment To Lessen Serial Cardiac Readmissions and Death) trial. J Am Coll Cardiol. 2009, 55:53-60. 10.1016/j.jacc.2009.02.095

21. McQuade CN, Mizus M, Wald JW, et al.: Brain-type natriuretic peptide and amino-terminal pro-brain-type natriuretic peptide discharge thresholds for acute decompensated heart failure: a systematic review. Ann Intern Med. 2017, 166:180-190. 10.7326/M16-1468

22. Huang YT, Tseng YT, Chu TW, et al.: N-terminal pro b-type natriuretic peptide (NT-pro-BNP) -based score can predict in-hospital mortality in patients with heart failure. Sci Rep. 2016, 6:29590.10.1038/srep29590

23. Tang WH, Girod JP, Lee MJ, et al.: Plasma B-type natriuretic peptide levels in ambulatory patients with established chronic symptomatic systolic heart failure. Circulation. 2003, 108:2964-2966. 10.1161/01.CIR.0000106903.98196.B6

24. Ellery S, Pakrashi T, Paul V, et al.: Predicting mortality and rehospitalization in heart failure patients with home monitoring--the Home CARE pilot study. Clin Res Cardiol. 2006, 95:III2935. 10.1007/s00392-006-1306-6

25. Adamson PB, Smith AL, Abraham WT, et al.: Continuous autonomic assessment in patients with symptomatic heart failure: prognostic value of heart rate variability measured by an implanted cardiac resynchronization device. Circulation. 2004, 110:2389-2394. 10.1161/01.CIR.0000139841.42454.78

26. Merchant FM, Dec GW, Singh JP: Implantable sensors for heart failure. Circ Arrhythm Electrophysiol. 2010, 3:657-667. 10.1161/CIRCEP.110.959502

27. Magalski A, Adamson P, Gadler F, et al.: Continuous ambulatory right heart pressure measurements with an implantable hemodynamic monitor: a multicenter, 12-month followup study of patients with chronic heart failure. J Card Fail. 2002, 8:63-70. 10.1054/jcaf.2002.32373

28. Reynolds DW, Bartelt N, Taepke R, et al.: Measurement of pulmonary artery diastolic pressure from the right ventricle. J Am Coll Cardiol. 1995, 25:1176-1182.

29. Adamson PB, Magalski A, Braunschweig F, et al.: Ongoing right ventricular hemodynamics in heart failure: clinical value of measurements derived from an implantable monitoring system. J Am Coll Cardiol. 2003, 41:565-571. 10.1016/S0735-1097(02)02896-6

30. Bourge RC, Abraham WT, Adamson PB, et al.: Randomized controlled trial of an implantable continuous hemodynamic monitor in patients with advanced heart failure: the COMPASS-HF study. J Am Coll Cardiol. 2008, 51:1073-1079. 10.1016/j.jacc.2007.10.061

31. Adamson PB, Gold MR, Bennett T, et al.: Continuous hemodynamic monitoring in patients with mild to moderate heart failure: results of The Reducing Decompensation Events Utilizing Intracardiac Pressures in Patients With Chronic Heart Failure (REDUCEhf) trial. Congest Heart Fail. 2011, 17:248-254. 10.1111/j.1751-7133.2011.00247.x

32. Plicchi G, Marcelli E, Parlapiano M, et al.: PEA I and PEA II based implantable haemodynamic monitor: pre clinical studies in sheep. Europace. 2002, 4:49-54. 10.1053/eupc.2001.0204

33. Ritzema J, Troughton R, Melton I, et al.: Physician-directed patient self-management of left atrial pressure in advanced chronic heart failure. Circulation. 2010, 121:1086-1095.

10.1161/CIRCULATIONAHA.108.800490

34. Maurer MS, Adamson PB, Costanzo MR, et al.: Rationale and design of the left atrial pressure monitoring to optimize heart failure therapy study (LAPTOP-HF). J Card Fail. 2015, 21:479488. 10.1016/j.cardfail.2015.04.012

35. Verdejo HE, Castro PF, Concepción R, et al.: Comparison of a radiofrequency-based wireless pressure sensor to swan-ganz catheter and echocardiography for ambulatory assessment of pulmonary artery pressure in heart failure. J Am Coll Cardiol. 2007, 50:2375-2382. 10.1016/j.jacc.2007.06.061 
36. Adamson PB, Abraham WT, Aaron M, et al.: CHAMPION trial rationale and design: the longterm safety and clinical efficacy of a wireless pulmonary artery pressure monitoring system. J Card Fail. 2011, 17:3-10. 10.1016/j.cardfail.2010.08.002

37. Abraham WT, Adamson PB, Bourge RC, et al.: Wireless pulmonary artery haemodynamic monitoring in chronic heart failure: a randomised controlled trial. Lancet. 2011, 377:658-666. 10.1016/S0140-6736(11)60101-3

38. Costanzo MR, Adamson PB, Abraham WT, et al.: Diuretic use guided by a wireless implanted pulmonary artery pressure monitoring system in NYHA class III heart failure patients: observations from the CHAMPION trial. Circulation. 2012, 126:A19396.

39. Goldberg LR, Desai AS, Costanzo MR, et al.: Pressure for action: implantable pulmonary artery pressure sensor measurements alone beat clinical signs to guide prevention of heart failure hospitalizations. Heart Rhythm. 2015,

40. Weiner S, Abraham WT, Adamson PB, et al.: Effect of CRT on heart failure related hospitalizations in patients with reduced EF utilizing remote pulmonary artery pressures in the CHAMPION trial. Heart Rhythm. 2011, 8:S437. 10.1016/j.hrthm.2011.03.033

41. Adamson PB, Abraham WT, Stevenson L, et al.: Benefits of pulmonary artery pressure monitoring extend to reduction of all-cause rehospitalization. J Am Coll Cardiol. 2014, 63:A746 . 10.1016/S0735-1097(14)60746-4

42. Raina A, Abraham WT, Adamson PB, et al.: Limitations of right heart catheterization in the diagnosis and risk stratification of patients with pulmonary hypertension related to left heart disease: insights from a wireless pulmonary artery pressure monitoring system. J Heart Lung Transplant. 2015, 34:438-447. 10.1016/j.healun.2015.01.983

43. Alam A, Jermyn R, Joseph M, et al.: Improved quality of life scores and exercise capacity with remote pulmonary artery pressure monitoring in patients with chronic heart failure. J Am Coll Cardiol. 2016, 67:1299. 10.1016/S0735-1097(16)31300-6

44. Desai AS, Bhimaraj A, Bharmi R, et al.: Reduction in heart failure hospitalizations with ambulatory hemodynamic monitoring seen in clinical trials is maintained in the 'Real World'. J Am Coll Cardiol. 2017, S0735-1097:35978-8. 10.1016/j.jacc.2017.03.009

45. Feldman D, Naka Y, Cabuay B, et al.: A wireless hemodynamic pressure sensor before and after ventricular assist device placement: a sub-study of the CHAMPION trial. J Heart Lung Transplant. 2011, 30:S86. 10.1016/j.healun.2011.01.248 